\title{
FPGA based Implementation of Embedding and Decoding Architecture for Binary Watermark by Spread Spectrum Scheme in Spatial Domain
}

\author{
Sudip Ghosh, Somsubhra Talapatra, Navonil Chatterjee, Santi P Maity and Hafizur Rahaman
}

\begin{abstract}
With the increasing influence of digital network and communication, armour from perilous intrusion and corruption of information (e.g. image, video, speech etc) during transmission is a paramount issue. To protect the image and video from the unauthenticated access or tampering, watermarking is adopted as a solution. In this paper we have proposed a spatial domain Spread Spectrum (SS) watermarking scheme using binary watermark which effectively eliminates security problem while increasing robustness and enhancing perceptual quality of watermarked image. Hardware implementation of the proposed digital image watermarking algorithm using Field Programmable Gate Array (FPGA) has been accomplished.
\end{abstract}

Keywords--- FPGA, Binary Watermark, Spatial Domain, Spread Spectrum, Digital Image Watermarking

\section{INTRODUCTION}

$\mathrm{T}$ HE process of embedding information into a digital signal which may be used for secure communication between the sender and recipient is called Digital watermarking. The information may be embedded in the digital signal such asaudio, image or video [8]. These days the use of internet has become a part and parcel in man's life. Information in form of digital images and video can be send and receive through the internet. The ever increasing efficacy of the internet and the ease of availability of sophisticated software make it possible to manipulate the digital content. Therefore the requirement for protection of digital content against unauthorized replication and modification has become the area of concern. A Digital Rights Management(DRM) technique deals with the

The preliminary version of this work has been appeared in the proceedings of ICACC 2012 [13].

Sudip Ghosh, Assistant Professor, School of VLSI Technology, Bengal Engineering and Science University, Shibpur, Howrah, India. E-mail: sudip_etc@yahoo.co.in

Somsubhra Talapatra, Assistant Professor, School of VLSI Technology, Bengal Engineering and Science University, Shibpur, Howrah, India. E-mail: s_talapatra@rediffmail.com

Navonil Chatterjee, Project Faculty, SMDPII, Bengal Engineering and Science University, Shibpur, India. E-mail:navonilchatterjee@yahoo.in

Santi P Maity, Associate Professor, Department of Information Technology, Bengal Engineering and Science University, Shibpur, Howrah, India.E-mail: spmaity@yahoo.com

Hafizur Rahaman, Professor, School of VLSI Technology, Bengal Engineering and Science University, Shibpur, Howrah, India. E-mail: rahaman_h@yahoo.co.in

DOI: 10.9756/BIJAIP.3096 ownership rights of the digital content. Digital watermarking though not complete DRM mechanism can be utilized in DRM systems to prevent unauthorized access and undesirable manipulation, thereby assuring authentication[2],[3].

In Digital watermarking, information is embedded in the form of symbol, text or a number. In case of invisible watermark it must be ensured that the modification of the media, as a result of embedding information, is imperceptible. Depending on perceptibility, digital watermarking has been divided into two types- : (i) Visible Watermark (ii) Invisible Watermark [7]. Classification can also be made on the basis of whether or not any Transformation technique has been implemented, namely- (i) Spatial Domain Watermarking [1][12] (ii) Transform Domain Watermarking [5][16].

Information transmitted through perilous communication channels is prone to contamination with undesirable and pernicious data or information may be tampered. To prevent this, the concept of Pseudo random Noise sequence is widely used in the domain of image watermarking and in steganography. In order to ensure secure data communication through the insecure communication channels, it is therefore necessary to utilize some stern and effective mechanism. Spread Spectrum watermarking technique is such a mechanism where, the information is spread over a larger frequency so that during extraction the integrity of the information is not surrogated [4],[5]. Spread spectrum (SS) watermarking may be used in fragile and semi fragile watermarking by keeping the chip rate low [6]. The term chip rate indicates the number of cover signal's sample over which the watermark bit is spread [9].

The objective of this paper is to design VLSI architecture for the given image watermarking algorithm that caters to the need of media authentication as well as secure transfer of image[14][15]. Hardware implementation of digital watermarking [11] provides several advantages over its software counterpart in terms of less area requirement, low execution time, and less power consumption. In this paper, the architecture that has been created for the given watermarking algorithm, enables watermarking to be implemented instantaneously at the time of capturing the image rather than using a software procedure that calls for greater execution time. Architecture has also been developed of the proposed algorithm. Digital design of the embedding portion of the proposed algorithm using FPGA has been developed. The example of TV broadcast will highlight the significance where digital media is to be marked in real time and hardware is the 
only solution. Another example is the solution to establishing the chain of custody for forensic digital photographers [10].

The rest of the paper is organized as follows. Section II describes the proposed watermarking algorithm. Section III and IV gives the Analysis and Attacks of the proposed watermarking scheme. Section V and VI represents the VLSI architecture of the Encoder and Decoder of the proposed algorithm. Section VII depicts the Result of proposed method where the device utilization table gives the synthesis report while section VIII administers the Conclusion.

\section{Proposed Watermarking Algorithm}

The following two subsections describe different steps in watermark embedding and decoding process respectively. Here, Binary watermark is embedded directly to the pixel values of each block of the cover image using SS modulation. During decoding, watermark information is extracted using normalized correlation and the extraction of binary watermark is done using channel decoding and spatial bi-phase demodulation [13].

\subsection{Watermark Embedding}

The Spread spectrum (SS) watermarking using binary watermark in spatial domain is discussed in details in the following section. Different steps for watermark embedding are described as follows:

\section{Step1: Image Partitioning}

The cover image is taken as $F$, where $F=\left\{F_{i j}, 1 \leq i \leq F_{\text {length }}\right.$, $\left.1 \leq \mathrm{j} \leq \mathrm{F}_{\text {width }}\right\}$, while $\mathrm{F}_{\mathrm{ij}} \in\{0,1, \ldots, 255\}, \mathrm{F}_{\text {length }}$ is the image length and $\mathrm{F}_{\text {width }}$ the width of image. Now we partition the cover image into ( $\mathrm{m} \mathrm{x} \mathrm{m}$ ) blocks which is non-overlapping, where $m=4,8,16,32$ etc. Suppose we call them as $H_{i j}$, where ' $i$ ' is the number of rows and ' $\mathrm{j}$ ' is the number of columns.

\section{Step 2: Formation of Message Vector}

The message image is taken as $\mathrm{W}$, where $\mathrm{W}=\left\{\mathrm{W}_{\mathrm{ij}}, 1 \leq \mathrm{i}\right.$ $\left.\leq \mathrm{W}_{\text {length }}, 1 \leq \mathrm{j} \leq \mathrm{W}_{\text {width }}\right\}$, while $\mathrm{W}_{\mathrm{ij}} \in\{0,1\}, \mathrm{W}_{\text {length }}$ is the image length and $\mathrm{W}_{\text {width }}$ the width of image. We partition the watermark image into $(\mathrm{L} \times \mathrm{L})$ non overlapping blocks, where $\mathrm{L}=2,4,8,16$ etc. We call them as $\mathrm{Q}_{\mathrm{ij}}$.

\section{Step 3: Formation of Compare Bit}

The MSB plane of 2-D pixel values of $\mathrm{H}_{\mathrm{ij}}$ is converted to 1-D strings. This forms the string1. Another binary string, string2 is formed using the bit values of the binary watermark image. An extended binary string is made by incorporating redundancy (repeating each bit 16 times). Now, the set of the strings formed from the cover image and the watermark image are compared with one another. If there occurs more than $50 \%$ positional match of the bits in the above form matrix, a bit ' 1 ' is assigned for the string otherwise bit ' 0 '. Bit ' 1 ' indicates inphase condition of two strings while out of phase condition is denoted by bit ' 0 '.

\section{Step 4: Generation of PN Code}

We derive the vector $S_{\mathrm{LxL}}$ from the PN (Pseudo Noise) sequence generated from the polynomial defined for a particular image length over which the message would be embedded. $\mathrm{S}=\left\{\mathrm{s}_{1}, \mathrm{~s}_{2}, \mathrm{~s}_{3}, \ldots . ., \mathrm{s}_{\mathrm{LxL}}\right\}, \mathrm{s}_{\mathrm{i}} \in\{0,1\}$. The vector $\mathrm{Z}$ is created by $z_{i}=2 s_{j}-1$, where $z_{i} \in\{1,-1\}$. If there are equal numbers of zeroes and ones are present is $\mathrm{S}$ then the vector $\mathrm{Z}$ will be a vector with zero mean. We have to generate $4 \mathrm{PN}$ (Pseudo Noise) codes of length ( $\mathrm{n} \times \mathrm{n}$ ), where $\mathrm{n}=4,8,16$ etc.

\section{Step 5: Watermark Embedding}

We now embedded the cover image with the watermark image using the Spread Spectrum (SS) watermarking scheme. The rule is given as:

$$
\begin{array}{ll}
\mathrm{F}^{\mathrm{e}}=\mathrm{F}+\mathrm{KS} & \text { if } \mathrm{b}_{\mathrm{j}}={ }^{\prime} 0 \\
\mathrm{~F}^{\mathrm{e}}=\mathrm{F}-\mathrm{KS} & \text { if } \mathrm{b}_{\mathrm{j}}={ }^{\prime}{ }^{\prime}
\end{array}
$$

Where

$\mathrm{F}^{\mathrm{e}}=$ Embedded image in spatial domain.

$\mathrm{F}=$ Cover image.

$\mathrm{K}=$ Modulation Index.

$\mathrm{S}=\mathrm{PN}$ code.

$b_{j}=$ The compare bit formed in step 3 of the algorithm

The value of the modulation index is calculated through experimental result evaluation.

\subsection{Watermark Image Extraction \& Message Decoding}

The watermark recovery process requires the sets of PN matrices (S) that were used for data embedding. Different steps for watermark decoding are described as follows:

\section{Step 1: Image Partitioning}

The received image $\mathrm{R}$ may be tampered with noise for which the brightness of the image can vary. The received image $\mathrm{R}$ is partitioned into $8 \times 8$ non overlapping blocks, suppose $R^{*}{ }_{i j}$ where, $i$ is number of rows and $j$ is number of column.

\section{Step 2: Correlation Calculation}

Correlation values between the watermarked image matrix and each code pattern of the set (S) are calculated. We have a total of $\left(\mathrm{M}_{\mathrm{m}} \cdot \mathrm{N}_{\mathrm{m}}\right)$ (equal to the number of watermark bits) correlation values $\left(r_{i}\right)$ where $i=1,2 \ldots M_{m} \cdot N_{m}$.

The decision rule for the decoded watermark bit is as follows:

1. For $r_{i} \geq 0$, the extracted bit is 0

2. For $r_{i}<0$, the extracted bit is 1 .

Step 3: Substring Decoding

MSB plane of the individual blocks of watermarked image or its distorted version is picked up and a set of $1 \mathrm{D}$ string $\mathrm{P}_{\mathrm{i}}=$ $\left\{\mathrm{P}_{1}, \mathrm{P} 2, \mathrm{P} 3, \mathrm{P} 4\right\}$ is generated from the $8 \times 8$ block. Bi-phase demodulation scheme is used in this stage. Based on the value of the extracted bit in the decoded watermark, the string either remains unchanged (if detected bit is ' 1 ') or complemented (if detected bit is ' 0 ').

\section{Step 4: Message Decoding}

Each string obtained in the above process is used to get back the binary watermark (bit redundancy). Binary detection is then applied for each substring based on the majority decision rule i.e. if more than $50 \%$ symbols are ' 1 ' in a sub substring, decision for decoding is ' 1 ', otherwise ' 0 '. The embedded binary digits obtained from the substrings are then converted to the pixel (each pixel of watermark image is 
represented by single bit) and binary watermark image is extracted.

\section{ANALYSIS OF WATERMARKED IMAGE}

The analysis of the watermarked image and the extracted watermark is done on the basis of the image quality and the perceptibility of the image and the amount of intrusion due to watermarking in the watermarked image. Also the error calculation of the extracted binary watermark compared to the original watermark is taken into account. Last but not the least we analyzed the variation of modulation index along with image quality.

The Structural Similarity (SSIM) index is a method for measuring the similarity between two images. The Peak Signal to Noise Ratio (PSNR) is the ratio of the maximum signal power to the corrupting noise power. The Mean Absolute Error (MAE) is a signal fidelity measure where the goal is to compare two signals by providing a quantitative score that describes the degree of similarity/ fidelity or, conversely, the level of error/distortion between them. Universal Image
Quality Index (Q) is a characteristic [21] of an image that measures the diminution or distortion of the received image to the original one. The Normalized Correlation (NC) is used to measure the similarity between two images.

Table 1: The Value of the Retrieved Watermark Image with Original Binary Watermark of the Size $(64 \times 64)$.

\begin{tabular}{|c|c|c|c|c|c|}
\hline $\begin{array}{c}\text { Value } \\
\text { of K }\end{array}$ & PSNR & MSSIM & Q & NC & MAE \\
\hline 1 & 3.8416 & 0.1537 & 0.1602 & 0.1583 & 0.4128 \\
\hline 5 & 7.5382 & 0.5558 & 0.5778 & 0.601 & 0.1762 \\
\hline 10 & 11.7302 & 0.8086 & 0.8256 & 0.8369 & 0.0671 \\
\hline 15 & 16.4857 & 0.9371 & 0.9381 & 0.9432 & 0.0224 \\
\hline 20 & 21.3523 & 0.9797 & 0.9744 & 0.9814 & 0.0073 \\
\hline 25 & 26.1236 & 0.991 & 0.9855 & 0.9937 & 0.0024 \\
\hline 30 & 36.1236 & 0.9978 & 0.9946 & 0.9993 & 0.0002 \\
\hline 1 & 36.1236 & 0.9978 & 0.9946 & 0.9993 & 0.0002 \\
\hline
\end{tabular}

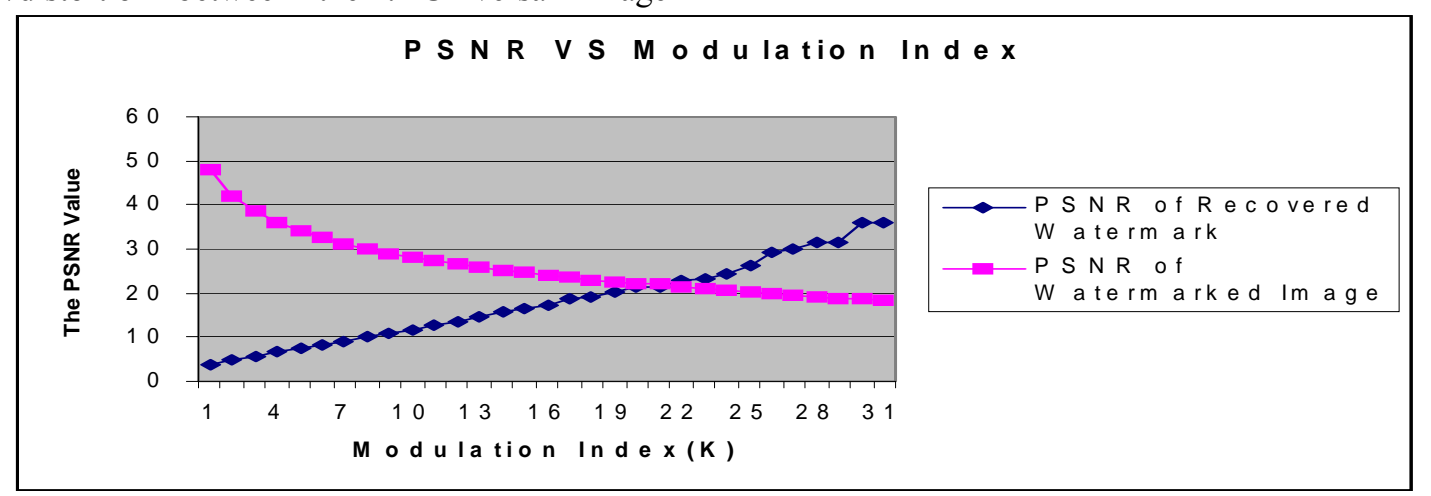

Figure 1: Graph Shows the Point of Intersection of PSNR of Watermark and Embedded Image. The Graph gives Optimized Value of Modulation Index (K)

Table 2: The Values of the Embedded Image with the Cover Image. Here the Cover is of Size (256 x 256)

\begin{tabular}{|r|l|r|l|l|l|}
\hline $\begin{array}{l}\text { Value } \\
\text { of K }\end{array}$ & PSNR & MSSIM & Q & NC & \multicolumn{1}{l|}{ MAE } \\
\hline 1 & 48.1309 & 0.998 & 0.9977 & 0.9997 & 0.9999 \\
\hline 5 & 34.1515 & 0.9552 & 0.9515 & 0.9928 & 4.9998 \\
\hline 10 & 28.1313 & 0.8588 & 0.8581 & 0.9724 & 9.9992 \\
\hline 15 & 24.61 & 0.7556 & 0.7622 & 0.9409 & 14.9975 \\
\hline 20 & 22.1122 & 0.6609 & 0.6738 & 0.9016 & 19.9939 \\
\hline 25 & 20.1753 & 0.577582 & 0.5948 & 0.8575 & 24.9874 \\
\hline 30 & 18.5942 & 0.5053 & 0.5252 & 0.8115 & 29.9142 \\
\hline 31 & 18.31 & 0.4921 & 0.5124 & 0.8022 & 30.9706 \\
\hline
\end{tabular}

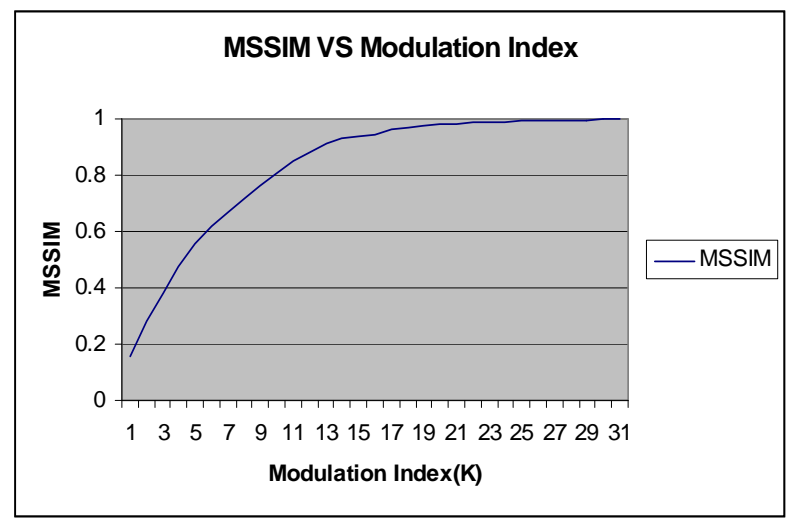

Figure 2: Graph shows that Mean Structural Similarity (MSSIM) Index Increases with Increase in Modulation Index (K)

From the above tables and graph we come to a conclusion that with the increase of the value of Modulation Index (K) the Quality of the retrieved binary watermark increases. But on the other hand if we increase $\mathrm{K}$, the perceptibility of the embedded image decreases. So we have to make a trade-off between the embedded image perceptibility and the quality of the recovered image. 


\section{ATTACK IMPLEMENTATION AND RECOVERY}

Various types of attack have been used during implementation of the algorithm to test its robustness to measure the error from the original to the extracted watermark the above mentioned methodologies are used. The common attacks along with JPEG compression are listed below in Table 3 and 4.

Table 3: The Value of the Retrieved Watermark Image with Original Binary Watermark of the Size (64 x 64)

\begin{tabular}{|l|c|r|l|l|l|}
\hline \multicolumn{1}{|c|}{ Attacks } & \multicolumn{1}{|c|}{ PSNR } & MSSIM & Q & \multicolumn{1}{l|}{ NC } & \multicolumn{1}{l|}{ MAE } \\
\hline Gaussian Filter & 36.124 & 0.999 & 0.998 & 0.9993 & 0.0002 \\
\hline Gaussian Noise & 29.134 & 0.998 & 0.994 & 0.9968 & 0.0012 \\
\hline $\begin{array}{l}\text { Salt and Pepper } \\
\text { Noise }\end{array}$ & 19.689 & 0.961 & 0.956 & 0.9729 & 0.0107 \\
\hline $\begin{array}{l}\text { Contrast } \\
\text { Stretching }\end{array}$ & 27.093 & 0.991 & 0.998 & 0.995 & 0.0019 \\
\hline Sickle Noise & 36.124 & 0.999 & 0.999 & 0.9993 & 0.0002 \\
\hline Sharpened & 33.113 & 0.999 & 0.998 & 0.9987 & 0.0004 \\
\hline
\end{tabular}

Table 4: The value of the Retrieved Watermark Image with Original Binary Watermark of the Size (64 x 64)

\begin{tabular}{|l|c|r|r|r|r|}
\hline $\begin{array}{l}\text { JPEG } \\
\text { attack }\end{array}$ & PSNR & MSSIM & Q & NC & \multicolumn{1}{l|}{ MAE } \\
\hline JPEG90 & Inf & 1 & 1 & 1 & 0 \\
\hline JPEG60 & 36.124 & 0.998 & 0.9946 & 0.9993 & 0.0002 \\
\hline JPEG50 & 36.124 & 0.998 & 0.9946 & 0.9993 & 0.0002 \\
\hline JPEG30 & 33.113 & 0.999 & 0.9986 & 0.9987 & 0.0004 \\
\hline JPEG20 & 20.326 & 0.973 & 0.9683 & 0.9765 & 0.0092 \\
\hline JPEG10 & 14.885 & 0.883 & 0.891 & 0.9179 & 0.0324 \\
\hline
\end{tabular}

The above shown tables error implementation and the results of each. The recovery of the watermark image under the influence of the above error is quite well. Here also we can see that the modulation index plays a vital role on the amount of recovery of the original watermark image and the error intrusion in the recovered image.

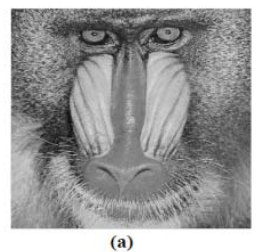

(a)

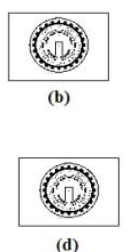

(d)

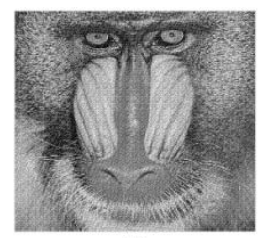

Figure 3(a) show the Cover Image of Size (256 x 256); Fig 3(b) the Binary Watermark Image of Size (64 x 64); Fig 3(c) the Embedded or Watermarked Image; Fig 3(d) the Recovered Watermark Image.

\section{Proposed VLSI ARCHITECTURE For ENCODER}

The architecture of the proposed watermark algorithm is shown in figure below. The main building blocks are as follows: (A) Controller (B) Memory Block (i) Memory Cover (ii) Memory Watermark (iii) Memory Compare (C) Assembly and Compare Block (D) Code block.

\subsection{Controller}

The functionality of the controller is governed by the configuration register. The configuration register is memory mapped means that CPU can write directly to the configuration register.

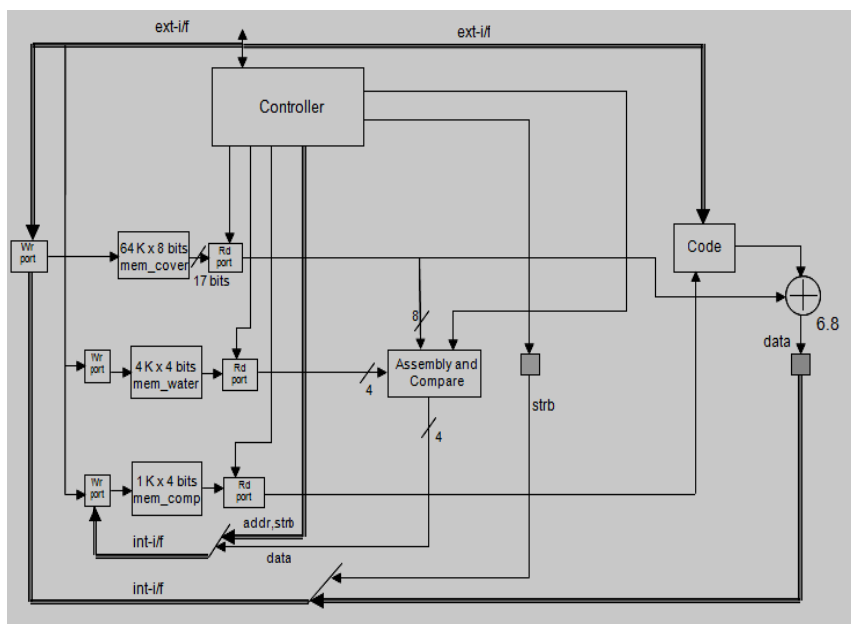

Figure 4: The Embedding Architecture of the Watermarking Algorithm.

The configuration is 3bit: $1^{\text {st }}$ Bit (B1) - Software Reset Bit, $2^{\text {nd }}$ Bit (B2) - Preprocess Bit, $3^{\text {rd }}$ Bit (B3) - Start Bit. These 3 bits are used to trigger 3 consecutive phases whose operation is described below.

In the Software reset phase internal register is reset to zero, where the reset is asynchronous. Here the images are downloaded in cover memory and watermark memory respectively. In Pre-process phase, b1, b2, b3, b4 (the four bits) set is calculated by reading $(8 \times 8)$ byte from memory cover and $(2 \times 2)$ bit are read from memory water in Consecutive cycles. 64 bit strings are formed as per the Algorithm in the Assembly and Compare block. Result is written in memory compare. In Start phase, pixels bytes are read out from Memory Cover sequentially. In this phase the embedding of the cover image is done by adding the pixel bytes with the output of the code block according to the algorithm. The embedded image is stored in the Memory Cover, which is extracted by the user after the process is completed.

\subsection{Memory Block}

The memory cover is $2^{16} \times 17$ bits memory which stores the cover image data of 8 bits. Next we have the memory water which is $2^{12} \mathrm{x} 4$ bit long. Here the watermark image is binary image which is represented by 4 bit. Lastly we have the Memory Compare which stores 4 bit compare data from the assembly and compare block. It is $2^{10} \mathrm{x}$ 4bit memory.

\subsection{Assembly and Compare Block}

A decoder is used where the select line is governed by 12 bit internal register. The two LSB bits of register are used as the select. Depending upon the register value and internal strobe each bit of watermark data is spawned 16 times and stored in water_64_out which is 6 bit long register. Now we compare the water_64_out with the cover_msb_64_dout which contains the MSB plane of the cover image $(8 \times 8)$ non 
overlapping block. The two 64 bit string water_64_out and cover msb 64 dout is compared using four comparator $(\mathrm{C} 1$, $\mathrm{C} 2, \overline{\mathrm{C}} 3, \overline{\mathrm{C}} 4)-16$ bit each. The output of the individual comparator is concatenated and the compare word is stored in Memory Embed.

\subsection{Code Block}

Code block implements $\pm(\mathrm{K} \mathrm{X} \mathrm{S})$ as used in the proposed algorithm given in fig. 6. The code block has four PN sequence generator S1, S2, S3, S4 and with the help of b1, b2, $\mathrm{b} 3$, b4, it generates a value of $\pm \mathrm{KS}$. $\mathrm{K}$ is a 10 bit register. MSB is the sign bit. Next bit is the integer bit and next 8 bits are for fraction. This $\mathrm{K}$ register is memory mapped to the external interface. $\pm \mathrm{S}$ is a 4 bit signed value and when multiplied with $\mathrm{K}$ register the output generates $(6+8)$ bit long word. The lower 8 bits are kept for fraction. If the operation is perfect, the MSB of the (6.8) is the extended sign bit. The bit next to MSB is the sign bit. These two bits should have identical values. Next four bits are for integer and last eight bits are for fraction. When this integer is added with (6.8) bit $\pm \mathrm{KS}$, decimal points has to be adjusted for the output of the transform.

\section{PROPOSED VLSI ARCHITECTURE FOR DECODER}

The decoding architecture of the proposed watermark algorithm is shown in figure 5 . The main building blocks are as follows: (A) Controller (B) Linear Feedback Shift Register (C) Memory (i) Watermark Memory (ii) Memory Embed (D) Correlator.

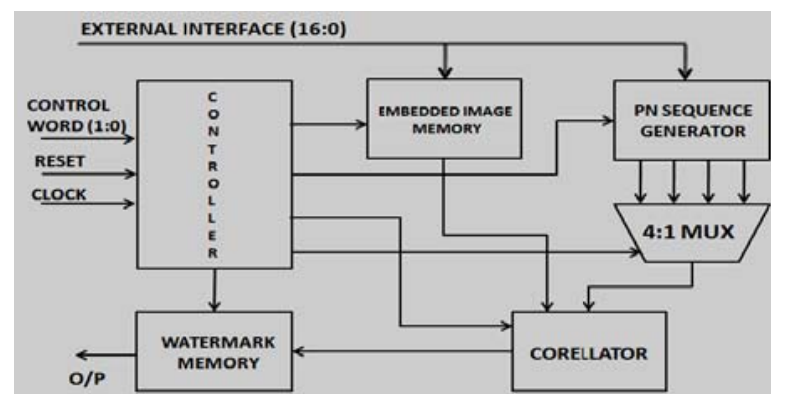

Figure 5: The Decoding Architecture of the Watermarking Algorithm.

\subsection{Controller}

The controller is controls the flow of the decoder in accordance to the control word. The control word is defined by a 2 bit register. The working and functionality of the control word is defined in the table 4 which breaks the functionality into different phases. These phases are triggered by the corresponding bit pattern of the control word. In Phase1 the four LFSRs are loaded with the seed value and the PN sequence is generated. The PN sequences formed are stored in four 6 bit registers. Along with this data (embedded data) is loaded in the Memory Embed is the same phase. In the next phase B1, B2, B3, B4 (the four bits) is calculated by performing correlation between the sub-blocks and respective PN sequence. The output of the correlator is stored in the Memory Watermark. Data is extracted from the Memory Watermark through the external bus interface 2 in Phase 3. The strobe signal is used for activation of the system. The architectural diagram of the decoder gives the detail of the control signals provided by the controller.

Table 5: The control Word is given below

\begin{tabular}{|c|c|l|}
\hline $\begin{array}{c}\text { Control } \\
\text { word }\end{array}$ & $\begin{array}{c}\text { State of } \\
\text { operation }\end{array}$ & \multicolumn{1}{|c|}{ Description } \\
\hline 01 & Phase I & $\begin{array}{l}\text { (i)LFSR set } \\
\text { (ii)Load data in Embed Memory }\end{array}$ \\
\hline 10 & Phase II & $\begin{array}{l}\text { (i) Decoding Operation } \\
\text { (ii) Resultant data is stored in } \\
\text { Watermark Memory }\end{array}$ \\
\hline 11 & Phase III & Data read from Watermark Memory \\
\hline
\end{tabular}

\subsection{Linear Feedback Shift Register}

The LFSR will be enabled by the controller in the Phase LFSR when it sets set seed $=$ ' 1 '. The seed is fed by the external interface by the user. The PN (Pseudo Noise sequence) is generated using the feedback mechanism. In the above stated algorithm we used PN sequence of length 64 bits. Depending on the polynomial the LFSR work in accordance to the taps to generate the PN sequence.

\subsection{Memory}

The Memory Embed is $2^{16}$ x 17 bits memory which stores the embedded image. Next we have the memory water which is $2^{12} \times 1$ bit long. The extracted binary watermark image data is stored in this memory.

\subsection{Correlator}

In this block the correlation between the embedded image and the PN sequence is calculated. The method by which the correlation is calculated as the $8 \times 8$ non-overlapping image block consisting of 64 elements is taken, each of which is multiplied with corresponding PN sequences (S1, S2, S3, and S4) which are 64 bit long. The outputs are added cumulatively and the result is divided by 64 . A comparator is used to calculate whether the outputs are greater than or less than the threshold, if it greater than the extracted bit is 0 else 1 . Now by using the majority logic we determine the extracted bit. If the number of ' 1 ' is more than or equal to eight then the extracted bit is ' 1 'else ' 0 '.

\section{RESULTS}

The proposed watermarking methodology is based on the combination of channel coding and spread spectrum technique. We consider a 1 bit/pixel of size $(64 \times 64)$ and 8 bits/pixel of size (256 x 256) binary images as watermark and the cover image respectively for experiment purpose. Now, the cover image is partitioned in $(8 \times 8)$ non-overlapping blocks and the watermark image is partitioned in $(2 \times 2)$ nonoverlapping blocks. The partitioning of the cover i.e. (8x8) and the watermark image followed by the conversion of the integer image data to binary was done with the help of MATLAB. With the binary image data for both the partitioned cover and the partitioned watermark image, we put them in the test bench as input cover and input watermark image. The same process in repeated for the decoding process. After the extraction of the decoded watermark image, the analysis and 
testing of the algorithm is done.

The synthesis of the watermark embedding have been implemented on Xilinx (ISE version8.1) based FPGA. We have chosen Virtex series of FPGA to fit the complexities of the design. The device used is xc2vp30-7ff896 for the implementation and the language used is VHDL. The behavioral simulation was done with Synopsys VCS-MX to verify the functionality of the design. A test bench is also written in VHDL to give the input vectors for the simulated program. The estimated power for the proposed architecture is calculated using Xpower of Xilinx 8.1, which comes around $1300 \mathrm{~mW}$ and the frequency of operation of the system, is $82.26 \mathrm{M} \mathrm{Hz}$.
Table 6: The Synthesis Results from Xilinx ISE 8.1

\begin{tabular}{|c|c|c|c|}
\hline \multicolumn{4}{|c|}{ Device Utilization Summary } \\
\hline Logic Utilization & Used & Available & Utilization \\
\hline Number of Slices & 959 & 13696 & $4 \%$ \\
\hline Number of Slice Flip Flops & 896 & 27392 & $1 \%$ \\
\hline Number of 4 input LUTs & 1669 & 27392 & $4 \%$ \\
\hline Number of Bonded IOBs & 39 & 416 & $13 \%$ \\
\hline Number of MULTS 18x18S & 1 & 136 & $0 \%$ \\
\hline Number of GCLKs & 1 & 16 & $6 \%$ \\
\hline
\end{tabular}

Table 7: Shows the Results of Extracted Watermark to the Original Watermark for Test Images $(256 \times 256)$ with $\mathrm{k}=20$

Table 7: The Results of Extracted Watermark to the Original Watermark for some Test Images

\begin{tabular}{|c|c|c|c|c|c|c|}
\hline \multirow[b]{2}{*}{$\begin{array}{l}\text { The Test } \\
\text { Images }\end{array}$} & \multicolumn{5}{|c|}{ Evaluation of the Extracted Watermark to the Original Watermark } & \multirow{2}{*}{$\begin{array}{c}\text { The } \\
\text { Extracted } \\
\text { Watermark }\end{array}$} \\
\hline & PSNR & MSSIM & Quality & $\begin{array}{l}\text { Normalized } \\
\text { Correlation }\end{array}$ & $\begin{array}{l}\text { Mean Absolute } \\
\text { Error }\end{array}$ & \\
\hline Airplane & 27.092700 & 0.94663 & 0.948434 & 0.993977 & 0.001953 & \\
\hline Baboon & 23.819110 & 0.853293 & 0.845930 & 0.987345 & 0.004150 & \\
\hline Barbara & 26.123599 & 0.918701 & 0.913132 & 0.992513 & 0.002441 & \\
\hline Boat & 26.5812 & 0.92605 & 0.92527 & 0.99323 & 0.0022 & \\
\hline Cameraman & 33.113300 & 0.988550 & 0.993745 & 0.998490 & 0.000488 & \\
\hline Lena & 27.0927 & 0.95958 & 0.96553 & 0.99396 & 0.00195 & \\
\hline Lighthouse & 23.1133 & 0.91431 & 0.9207 & 0.98502 & 0.00488 & \\
\hline Pepper & 36.1236 & 0.99739 & 0.99904 & 0.99925 & 0.00024 & \\
\hline
\end{tabular}

Table 8, Gives comparative study of VLSI Architectures of Image Watermarking Systems Proposed in the Literature which shows our Experimental Results have improvement.

Table 8: The comparative Study of VLSI Architectures of Image Watermarking Systems Proposed in the Literature

\begin{tabular}{|c|c|c|c|c|c|}
\hline Research Work & $\begin{array}{l}\text { Design } \\
\text { Type }\end{array}$ & Watermarking Type & $\begin{array}{l}\text { Multimedia } \\
\text { Object }\end{array}$ & Working Domain & $\begin{array}{c}\text { Chip } \\
\text { Statistics }\end{array}$ \\
\hline $\begin{array}{l}\text { Mohanty et. al } \\
{[14],[20]}\end{array}$ & $\begin{array}{c}\text { FPGA } \\
\text { platform }\end{array}$ & Invisible & Image & Spatial & $\begin{array}{c}\text { Xilinx, Virtex Series } \\
\text { XCV50-BG256-6, 50.398MHz }\end{array}$ \\
\hline $\begin{array}{l}\text { Ghosh et. al } \\
{[17],[18]}\end{array}$ & $\begin{array}{l}\text { FPGA } \\
\text { platform }\end{array}$ & $\begin{array}{c}\text { Invisible } \\
\text { (Binary Watermark) }\end{array}$ & Image & $\begin{array}{c}\text { Transform } \\
\text { (Walsh Hadamard } \\
\text { Transform) }\end{array}$ & $\begin{array}{c}\text { Xilinx 14.1, Virtex } 4 \\
\text { XC4vlx200-11ff1513, } \\
\text { Max. Frequency 90.131 MHz }\end{array}$ \\
\hline $\begin{array}{c}\text { Ghosh et. al } \\
{[16]}\end{array}$ & $\begin{array}{c}\text { FPGA } \\
\text { platform }\end{array}$ & $\begin{array}{c}\text { Invisible } \\
\text { (Binary Watermark) }\end{array}$ & Image & $\begin{array}{c}\text { Spatial and } \\
\text { Transform (Dual } \\
\text { mode) }\end{array}$ & $\begin{array}{c}\text { Xilinx 14.1, Virtex } 4 \\
\text { XC4vlx200-11ff1513, } \\
\text { Max. Frequency } 90.131 \mathrm{MHz}\end{array}$ \\
\hline Maity et. al [19] & $\begin{array}{l}\text { FPGA } \\
\text { platform }\end{array}$ & Invisible & Image & $\begin{array}{l}\text { Spatial bi-phase } \\
\text { Modulation based }\end{array}$ & $\begin{array}{c}\text { Xilinx,Spartan Series } \\
\text { XCS-05; CLB count }=85 \\
\text { Max. Frequency }=80 \mathrm{MHz}\end{array}$ \\
\hline $\begin{array}{c}\text { Ghosh et. al } \\
\text { [1] }\end{array}$ & $\begin{array}{c}\text { FPGA } \\
\text { platform }\end{array}$ & $\begin{array}{c}\text { Invisible } \\
\text { (Gray-scale } \\
\text { Watermark) }\end{array}$ & Image & Spatial & $\begin{array}{c}\text { Xilinx(ISE version 8.2i),Virtex } 4 \\
\text { xc4vlx25-10ff676 } \\
\text { Maximum Frequency: } 219.542 \mathrm{MHz}\end{array}$ \\
\hline $\begin{array}{c}\text { Ghosh et.al } \\
{[12]}\end{array}$ & $\begin{array}{c}\text { FPGA } \\
\text { platform }\end{array}$ & $\begin{array}{l}\text { Invisible } \\
\text { (Gray-scale } \\
\text { Watermark) }\end{array}$ & Image & Spatial & $\begin{array}{c}\text { Xilinx, Virtex Series } \\
\text { xc2vp30-7ff896 } \\
\text { power consumption is } 650 \mathrm{mw} \\
\text { maximum frequency is } 82.204 \mathrm{MHz} \text {. }\end{array}$ \\
\hline $\begin{array}{c}\text { Ghosh et.al } \\
{[13]}\end{array}$ & $\begin{array}{c}\text { FPGA } \\
\text { platform }\end{array}$ & $\begin{array}{c}\text { Invisible } \\
\text { (Binary Watermark) }\end{array}$ & Image & Spatial & $\begin{array}{c}\text { Xilinx (ISE version8.1) } \\
\text { Virtex series }(x c 2 \mathrm{vp} 30-7 \mathrm{ff} 896) \\
\text { Frequency of operation of the is } 82.26 \mathrm{MHz} \text {. } \\
\text { Estimated Power }=650 \mathrm{~mW}\end{array}$ \\
\hline
\end{tabular}




\section{CONCLUSIONS}

In this paper we have proposed a spatial domain image watermarking scheme using binary watermark by combined use of channel coding and spread spectrum modulation. The algorithm is simple with low computation cost and can be easily implemented in hardware. Digital design of the proposed algorithm using FPGA has been developed and thus makes it suitable for real time authentication as well as secured communication. The analysis of the watermark image shows with the increase of the value of Modulation Index (K) the Quality of the retrieved binary watermark increases but the perceptual quality of the embedded image degrades. Hence there is a trade off between these two parameters. The VLSI architecture of the Encoder and Decoder had been implemented with Xilinx based Virtex 2pro FPGA and the observations are mentioned.

\section{ACKNOWLEDGMENT}

This work has been partially supported by the SMDP II project, DIT, MCIT, Government of India.

\section{REFERENCES}

[1] Sudip Ghosh, Pranab Ray, Santi P Maity and Hafizur Rahaman, "Spread Spectrum Image Watermarking with Digital Design", IEEE International Advance Computing Conference (IACC 2009), Pp.868-873, 2009.

[2] Special issue on copyright and privacy protection, IEEE Journal on Selected Areas in Communication (JSCA), 16(4), May 1998.

[3] Special issue on enabling security technologies for digital right management, Proceedings of IEEE, 92(6), June 2004.

[4] Rui Bao, Tianqi Zhang, Fangqing Tan and Y.E. Wang, "Semi-fragile watermarking algorithm of color image based on Slant Transform and channel coding", Image and Signal Processing (CISP), 2011 4th International Congress on, On page(s): 1039 - 1043 Vol. 2, Pp.15-17 Oct. 2011

[5] I.J. Cox, J. Kilian, T. Leighton, and T. Shamoon, Secure spread spectrum watermarking for multimedia, IEEE Transaction on Image Processing , 6 (12), Pp. 1673-1687, 1997.

[6] S.P. Maity, M.K. Kundu and T.S. Das, "Robust SS Watermarking with improved capacity", Pattern Recognition Letters "Advances in Visual Information Processing", Elsevier, 28, Pp. 350-357, 2007.

[7] S.P. Maity and M.K. Kundu, "A blind CDMA image watermarking scheme in wavelet domain," Proc. Of IEEE Int. Conf. on Image Proc., Singapore, Pp. 2633-2636, 2004.

[8] N.J. Mathai, D. Kundur, and A. Sheikholeslami, Hardware implementation perspectives of digital video watermarking algorithms, IEEE Transaction on Signal Processing, Vol. 51, Pp. 925- 938, April 2003.

[9] S.P. Maity, A. Banerjee, A. and M. K. Kundu, VLSI design of Spread Spectrum Image Watermarking-13th National Conference on communications (NCC-2007), Indian Institute of Technology, Kanpur, India, January 26-28, Pp. 251-257, 2007.

[10] S.P. Maity , M.K. Kundu and Mrinal K Mandal "Performance Improvement in Spread Spectrum Watermarking via M-band Wavelets and N-ary Modulation"IET International Conference Visual Informal Engineering(VIE 2006), Pp. 35 -40,2006.

[11] E. Kougianos, S.P. Mohanty, and R.N. Mahapatra, "Hardware Assisted Watermarking for Multimedia", Special Issue on Circuits and Systems for Real-Time Security and Copyright Protection of Multimedia, Elsevier International Journal on Computers and Electrical Engineering (IJCEE), Vol. 35, No. 2, Pp. 339-358. March, 2009,

[12] Sudip Ghosh, Somsubhra Talapatra, Debasish Mondal, Navonil Chatterjee, Hafizur Rahaman and Santi P Maity, "VLSI Architecture for Spatial Domain Spread Spectrum Image Watermarking using GrayScale Watermark", in 16th International Symposium on VLSI Design and Test (VDAT, 2012) from 1-4 July 2012 at Bengal Engineering and Science University, Shibpur, India. Pp. 375-376 (Springer, LNCS),2012.

[13] Sudip Ghosh, Somsubhra Talapatra, Debasish Mondal, Navonil Chatterjee, Hafizur Rahaman and Santi P Maity, "VLSI Architecture for
Spread Spectrum Image Watermarking using BinaryWatermark" in IEEE, International Conference on Advances in Computing and Communications(ICACC), from 9-11 August 2012 at Rajagiri School of Engineering \& Technology, Cochin, Kerala, India, Pp.166-169, 2012.

[14] Saraju P. Mohanty, C. Renuka Kumara and Sridhara Nayak, "FPGA based implementation of an invisible-robust image watermarking encoder" Intelligent Information Technology, Lecture Notes in Computer Science Vol. 3356, Pp. 344-353, 2005.

[15] A. Basu, T.S. Das, S. Maiti, N. Islam and S.K. Sarkar, "FPGA based implementation of robust spatial domain image watermarking algorithm", 4th International Conference on Computers and Devices for Communication, CODEC ,Pp 1-4, 2009

[16] Sudip Ghosh, Somsubhra Talapatra, Jayasree Sharma, Navonil Chatterjee, Hafizur Rahaman and Santi P Maity, "Dual Mode VLSI Architecture for Spread Spectrum Image Watermarking using Binary Watermark" in 2nd International Conference on Communication, Computing \& Security (ICCCS-2012) from 6-8 October 2012 at National Institute of Technology Rourkela, India.Pp.784-791, 2012.

[17] Sudip Ghosh, Somsubhra Talapatra, Santi P Maity and Hafizur Rahaman "A Novel VLSI Architecture for Walsh-Hadamard Transform" in IEEE 2nd Asia Symposium on Quality Electronic Design (ASQED-2010) from 3-4 August, 2010 at Penang, Malaysia. Pp. 146 - 150, 2010.

[18] Sudip Ghosh, Somsubhra Talapatra, Sudipta Chakraborty, Navonil Chatterjee, Hafizur Rahaman and Santi P Maity, "VLSI Architecture for Spread Spectrum Image Watermarking in Walsh-Hadamard Transform Domain using Binary Watermark" in 3rd IEEE International Conference on Computer and Communication Technology ( ICCCT 2012) from 23-25 November 2012, at Motilal Nehru National Institute of Technology(MNNIT), Allahabad, India. Pp. 233-238, 2012.

[19] S.P. Maity, A. Banerjee and M.K. Kundu"An Image-inImagecommunication scheme and VLSI implementation using FPGA",INDICON 2004, IIT Kharagpur, India. Pp.6-11, 2004.

[20] S.P. Mohanty, E. Kougianos and N. Ranganathan, "VLSI architecture and chip for combined invisible robust and fragile watermarking", IET Computers \& Digital Techniques (CDT) 1(5) Pp. 600-611, 2007.

[21] Z. Wang, A.C. Bovik, H.R. Sheikh and E.P. Simoncelli, "Image quality assessment: From error visibility to structural similarity,"IEEE Transactions on Image Processing, Vol. 13, No. 4, Pp. 600-612, Apr. 2004.

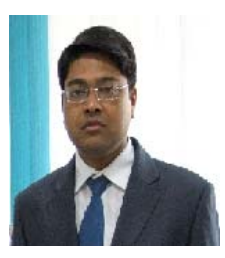

Sudip Ghosh was born at Kolkata, West Bengal, India in the year of 1977. He received his B.E. in Electronics and Communication Engineering from Sikkim Manipal Institute of Technology, Sikkim, India in the year 2001 and M.S. in VLSI CAD from Manipal Centre of Information Science (MCIS),Manipal, India, in the year of 2005.He completed his B.E. project at VECC(Variable Energy Cyclotron Centre ), kolkata(under DAE (Department of Atomic Energy, Govt. of India). After his B.E. he worked at Saha Institute of Nuclear Physics, Kolkata,India at Microelectronics division (Govt. of India) and also done his M.S. project at Indian Statistical Institute (ISI), Kolkata at Advanced Computing And Microelectronics Unit(ACMU) (Govt. of India).He is a member of CSI(Computer Society of India) and VSI (VLSI Society of India). Currently he is working as an Assistant Professor at School of VLSI Technology, Bengal Engineering And Science University, Shibpur.(BESUS), Howrah, India and also actively doing his research at BESUS. His area of specialization includes Synthesis of Digital Systems, Digital VLSI architectures, VLSI Testing and Verification and Digital Watermarking of Image and Video signals.(E-mail: sudip_etc@yahoo.co.in)

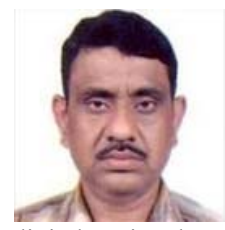

Santi P. Maity is working as Associate Professor in the Department of Information Technology, BESU, Shibpur, India. During January, 2009 to July, 2009 and February, 2011 to July, 2011 he did post-doctoral research work in Laboratoire des Signaux et Systems, Supelec, France. His research areas include digital image watermarking, multi-user detection in CDMA, digital signal processing, digital wireless communication, VLSI watermarking. He has contributed about 100 research papers. 


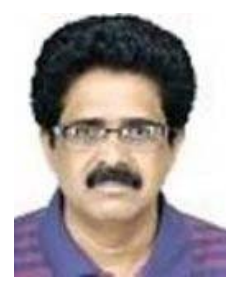

Hafizur Rahaman (IEEE SM'10) received the Bachelor of Electrical Engineering degree from Calcutta University, India, in 1986, the Master degree in Electrical Engineering and Ph.D. (Computer SC. \& Engg.) degree from the Jadavpur University, Calcutta, India, in 1988 and 2003 respectively. He served CMPD Institute (R \& D organization), India, from 1988 to 1995. He served as a faculty member at India Institute of Information Technology-Calcutta (IIIT-C), India, from 1995 to 2003. Since 2003, Dr. Rahaman has been on the faculty of the Bengal Engineering and Science University, Shibpur, India, where he is full professor. Dr. Rahaman has contributed to very large scale integrated computer-aided design and test, design and test of Micro-fluidic biochips and emerging nanotechnologies with major publications in journals and conferences, spanning more than 15 years. He has published more than 140 research articles in archival journals and refereed conference proceedings. Dr. Rahaman visited as Post Doctoral Research Fellow under EPSARC Grant at the department of Computer Science, Bristol University, UK, during 20062007. During 2008-2009, Dr. Rahaman received Royal Society International Fellowship Award to carry out one year advanced research in the Design and Verification Division of Computer Science Department, University of Bristol, United Kingdom. He leads the VLSI design and test group at the Bengal Engineering and Science University, Shibpur, India. He is the principal coordinator of the department of Information Technology (DIT), MCIT, Govt. of India funded SMDP-II research project at Bengal Engineering and Science University, Shibpur, India.

Dr. Rahaman is a Member of the VLSI Society of India (VSI), the IEEE, the IEEE Computer Society, and ACM Sigda. He served on the conference committees of the International Conference on VLSI Design, the VLSI Design and Test Workshop (VDAT), Asian Test symposium (2005), ISED (2010, 2012). 